\title{
Phase Space Distribution of Particles Near an Isolated Difference Resonance
}

\author{
Jicong Shi, Yunxiang Huang and Shoroku Ohnuma \\ Department of Physics, University of Houston, Houston, Txi7204-5504
}

\begin{abstract}
We have studied the phase space distribution of beam particles near an isolated difference resonance. The numerical simulation has shown that the distribution can quickly settle down to a final state if its initial state is Gaussian. The final state can be treated as a stationary state as long as the high order fluctuation can be neglected. From the Liouville equation, the emittances of beam particles are calculated approximately. There is a good agreement between analytical and numerical results.
\end{abstract}

\section{Introduction}

In the system with two degrees of freedom and time dependence, an isolated difference resonance does not lead to an instability, i.e., the motion is always confined in both $x$ and $y$ directions. ${ }^{1,2}$ For a beam of particles, therefore, the emittances in two directions are bounded. Due to the coupling, however, the energy could be transferred from one direction to the other. As a result, an initially very small emittance in one direction could grow to a large value. Even though this growth may be acceptable as far as the stability is concerned, it could influence the particle distribution of the beam.

Consider an isolated difference resonance of the form

$$
(2 p) \nu_{x}-(2 q) \nu_{y}=n+\gamma
$$

where $2 p$ and $2 q$ are positive integers with $2 p+2 q=2, n \geq$ 0 an integer, and $|\gamma| \ll 1 . \nu_{x}$ and $\nu_{y}$ are the horizontal and vertical tunes. In the single resonance approximation, the Hamiltonian in terms of the action-angle variables $(I, \phi)$ can be written as

$$
H=\nu_{x} I_{x}+\nu_{y} I_{y}-\epsilon I_{x}^{p} I_{y}^{q} \cos \left(2 p \phi_{x}-2 q \phi_{y}+n \theta\right) .
$$

Using the canonical transformation

$$
S\left(\phi_{x}, J_{x}, \phi_{y}, J_{y}\right)=\left(\phi_{x}-\nu_{x}^{0} \theta\right) J_{x}+\left(\phi_{y}-\nu_{y}^{0} \theta\right) J_{y},
$$

where $\nu_{x, y}^{0}$ are the tunes of the exact resonance, $(2 p) \nu_{x}^{0}-$ $(2 q) \nu_{y}^{0}=n$ and $\nu_{x, y}=\nu_{x, y}^{0}+\gamma_{x, y}$, we obtain a "time" independent Hamiltonian,

$$
K=\gamma_{x} I_{x}+\gamma_{y} I_{y}-\epsilon I_{x}^{p} I_{y}^{q} \cos \left(2 p \psi_{x}-2 q \psi_{y}\right),
$$

${ }^{1}$ Supported by the U.S. Department of Ene. gy under grant DEFG05-87ER40374. with $\psi_{x, y} \equiv \phi_{x, y}-\nu_{x, y}^{0} \theta$. Since the dependence of $k$ on angle variables is only through $2 p \psi_{x}-2 q \psi_{y}, I_{x} /(2 p)+$ $I_{y} /(2 q)$ is another constant of motion besides $K$.

The behavior of a beam can be described by the phase space distribution of particles $f\left(I_{x}, \psi_{x}, I_{y}, \psi_{y}, \theta\right)$, of which the time evolution is governed by the Liouville equat:on ${ }^{3}$,

$$
\frac{\partial f}{\partial \theta}=\{K, f\}
$$

where \{\} is the Poisson bracket. For the Hamiltonian (4), this equation becomes

$$
\begin{gathered}
L f=\epsilon\left\{I_{x}^{p} I_{y}^{q} \sin \left(2 p \dot{\psi}_{x}-2 q \psi_{y}\right)\left(2 p \frac{\partial}{\partial T_{x}}-2 q \frac{\partial}{\partial T_{y}}\right)\right. \\
\left.+\cos \left(2 p \psi_{z}-2 q \psi_{y}\right)\left(p I_{x}^{p-1} I_{y}^{q} \frac{\partial}{\partial \psi_{x}}+q I_{x}^{p} I_{y}^{q-1} \frac{\partial}{\partial \psi_{y}}\right)\right\} f
\end{gathered}
$$

where

$$
L=\frac{\partial}{\partial \theta}+\gamma_{x} \frac{\partial}{\partial \psi_{x}}+\gamma_{y} \frac{\partial}{\partial \psi_{y}}
$$

is an integrable operator. Since the Liouville operator $\mathcal{L}=i\{K$,$\} has only real eigenvalues, { }^{3}$ the solution of the Liouville equation will not settle into a unique equilibrium state. The final state of the Liouville equation depends on the initial distribution. It is easy to see that the angle independent stationary state of Eq.(6) is $f=f\left(I_{x} / 2 p+I_{y} / 2 q\right)$ which is an arbitrary function of $I_{x} / 2 p+I_{y} / 2 q$. Since $I_{x} / 2 p+I_{y} / 2 q$ is a constant of motion, the mean actions, which correspond to the emittances in two directions, satisfy

$$
\left\langle I_{x}\right\rangle / 2 p+\left\langle I_{y}\right\rangle / 2 q=\text { constant } .
$$

In the following, we present a coupled sextupole resonance: $2 p=1,2 q=2$ and $n=2$. The treatments is, however, applicable to any $p, q$ and $n$ values.

\section{Numerical simulation}

To understand the evolution of particle distribution, we have tracked numericaliy an ensemble of particles in il test lattice with four sextupoles placed symmetrically. Define $\xi_{x} \equiv \beta_{0}^{-\frac{1}{2}} x, \xi_{y} \equiv \beta_{0}^{-\frac{1}{2}} y, \eta_{x} \equiv \beta_{0}^{-\frac{1}{2}} d x / d 0$, and $\eta_{x} \equiv \beta_{0}^{-\frac{1}{2}} d y / d \theta$. At each sextupole, $\xi_{x, y}$ are unchinged but

$$
\begin{aligned}
& \Delta \eta_{x}=-s \beta_{0}^{\frac{3}{2}}\left(\xi_{x}^{2}-\xi_{y}^{2}\right) \\
& \Delta \eta_{y}=2 s \beta_{0}^{\frac{3}{3}} \xi_{x} \xi_{y}
\end{aligned} \text { M HSTER }
$$




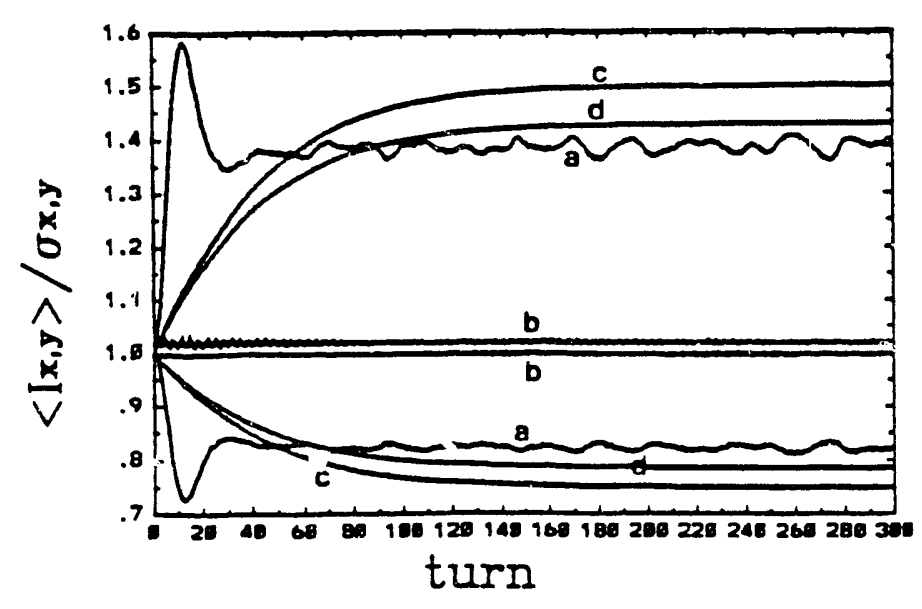

Figure 1: The evolution of the emittances from the numerical tracking of 1000 particles, $(a)$ and $(b)$, and from the equations of the monents, $(c)$ and $(d)$. The initial state is a Gaussian distribution with $\sigma_{x}=\sigma_{y}=0.5 \times 10^{-3} \mathrm{~mm}$, and $s_{0}=0.05 \mathrm{~m}^{-2}, \beta_{0}=100 \mathrm{~m}$. In each curve the upper branch is $\left\langle I_{y}\right\rangle / \sigma_{y}$ and the lower one $\left\langle I_{x}\right\rangle / \sigma_{x} .(a)$ is on the resonance with $\nu_{x}=1.65$ and $\nu_{y}=1.825$. (b) is far away from the resonance with $\nu_{x}=1.70$ and $\nu_{y}=1.65$. (c) and $(d)$ have the same conditions as $(a)$, but $(c)$ is calculated from the map (20) and (d) is calculated from the modified version of the map (21) in which Eq.(24) instead of Eq.(13) is used.

where $s=s_{0}$ at $\theta=m \pi$ and $s=-s_{0}$ at $\theta=(m+1 / 2) \pi$ for $m \geq 0$. Between sextupoles, it is a linear rotation

$$
\left[\begin{array}{cc}
c_{x, y} & s_{x, y} \\
-s_{x, y} & c_{x, y}
\end{array}\right] \cdot\left[\begin{array}{l}
\xi_{x, y} \\
\eta_{x, y}
\end{array}\right] \rightarrow\left[\begin{array}{l}
\xi_{x, y} \\
\eta_{x, y}
\end{array}\right]
$$

where $c_{x, y}=\cos \left(2 \pi \nu_{x, y} / 4\right)$ and $s_{x, y}=\sin \left(2 \pi \nu_{x, y} / 4\right)$. The Hamiltonian of this system can be written as

$$
\begin{aligned}
H= & \frac{1}{2} \nu_{x}\left(\xi_{x}^{2}+\eta_{x}^{2}\right)+\frac{1}{2} \nu_{y}\left(\xi_{y}^{2}+\eta_{y}^{2}\right) \\
& +\frac{1}{3} s_{0} \beta_{0}^{\frac{3}{2}} b(\theta)\left(\xi_{x}^{3}-3 \xi_{x} \xi_{y}^{2}\right)
\end{aligned}
$$

where $b(\theta)=\delta(\theta)-\delta\left(\theta-\frac{\pi}{2}\right)+\delta(\theta-\pi)-\delta\left(\theta-\frac{3 \pi}{2}\right)$. In the single resonance approximation, this Hamiltonian can be easily reduced to the Hamiltonian (2) with $2 I_{x, y}=\xi_{x, y}^{2}+$ $\eta_{x, y}^{2}$ and $2 p=1,2 q=2, n=2$. The initial particle distribution of the beam is assumed to be Gaussian

$$
f\left(\xi_{x}, \eta_{x}, \xi_{y}, \eta_{y}, 0\right) \sim \exp \left(-\frac{\xi_{x}^{2}+\eta_{x}^{2}}{2 \sigma_{x}}-\frac{\xi_{y}^{2}+\eta_{y}^{2}}{2 \sigma_{y}}\right) .
$$

When the operating point is very close to the resonance $\left(\gamma_{x, y} \ll 1\right)$, it has been found out that the distribution function quickly settles down to a final state. Exiept for the small fluctuation, the mean actions (emittances) in the final state achieve the stationary values. The curve $(a)$ in Fig. 1 is an example of "on-the-resonance" case, i.e., $\gamma_{x, y}=$

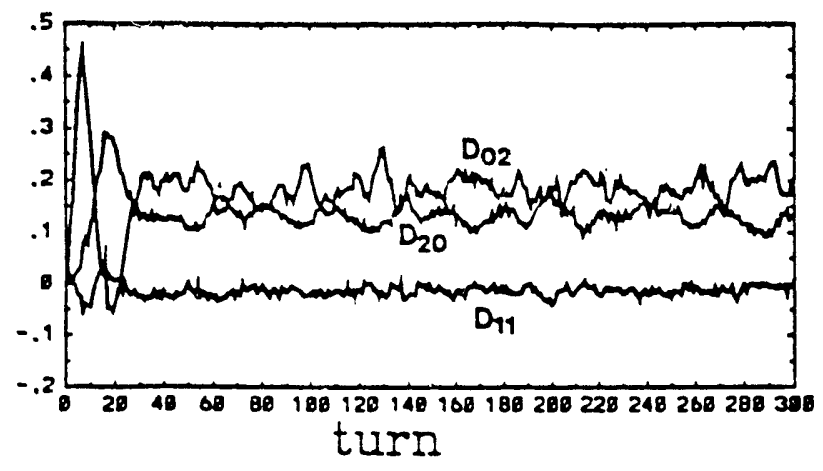

Figure 2: Relative fluctuation of $I_{x, y}$ of curve $(a)$ in Fig.1. $D_{11}=\left(\left\langle I_{x} I_{y}\right\rangle-\left\langle I_{x}\right\rangle\left\langle I_{y}\right\rangle\right) /\left\langle I_{x}\right\rangle\left\langle I_{y}\right\rangle, D_{02}=\left(\left\langle I_{x}^{2}\right\rangle-\right.$ $\left.2\left\langle I_{x}\right\rangle^{2}\right) /\left\langle I_{x}\right\rangle^{2}$, and $D_{20}=\left(\left\langle I_{y}^{2}\right\rangle-2\left\langle I_{y}\right\rangle^{2}\right) /\left\langle I_{y}\right\rangle^{2}$.

0 . To examine the final distribution, we computed the deviation of the second order moments of the actions from the relation

$$
\left\langle\Delta I_{\alpha} \Delta I_{\beta}\right\rangle=\left\langle I_{\alpha} I_{\beta}\right\rangle-\left\langle I_{\alpha}\right\rangle\left\langle I_{\beta}\right\rangle=\delta_{\alpha \beta}\left\langle I_{\alpha}\right\rangle\left\langle I_{\beta}\right\rangle
$$

of the Gaussian distribution where $\delta_{\alpha \beta}$ is the Krone:ker $\delta$-symbol. It is shown in Fig. 2 that this deviation is very small except in the transient period. Thus the final state could be treated as a stationary Gaussian distribution as long as the high order fluctuation can be neglected. When the operating point is far away from the resonance $\left(\gamma_{x, y} \gg\right.$ 1), the motion of two directions is decoupled so that the particle distribution remains unchanged as shown by the curve (b) in Fig.1.

\section{Equations of the moments}

Using equations of the moments, we can find the emittances approximately, but because of the sinusoidal dependence of the angle variables, it is impossible to obtain the equations of the moments directly from $\mathrm{Eq} .(6)$. To avoid this difficulty, we define

$$
E_{i j} \equiv \int I_{x}^{i} I_{y}^{j} f d I_{x} d I_{y}
$$

The $(i, j)$ order moment is then

$$
\left\langle I_{x}^{i} I_{y}^{j}\right\rangle=\int E_{i j} d \dot{\psi}_{x} d \psi_{y}^{\prime} .
$$

Multiplying Eq.(6) by $I_{x}^{i} I_{y}^{j}$ and integrating it over $l_{x}$ and $I_{y}$, we obtain a system of infinite numbers of equations to be satisfied by $E_{i j}$. Generally, the equation for $E_{i j}$ involves higher order terms $E_{i+\frac{1}{3} j}$ and $E_{i-\frac{1}{2} j+1}$. Thie first three equations, which are relevant to the evolution of $\left\langle I_{x}\right\rangle$, are

$$
L E_{10}=\epsilon\left\{\left[2 \sin (\Delta \psi)+\cos (\Delta \psi) \frac{\partial}{\partial \dot{\psi}_{\mathrm{y}}}\right] E_{\frac{z}{3} 0}\right.
$$




$$
\begin{aligned}
&+\left.\frac{1}{2}\left[-3 \sin (\Delta \psi)+\cos (\Delta \psi) \frac{\partial}{\partial \psi_{x}}\right] E_{\frac{1}{\partial 1}}\right\} \\
& L E_{\frac{3}{2} 0}=\epsilon\left\{\left[2 \sin (\Delta \psi)+\cos (\Delta \psi) \frac{\partial}{\partial \psi_{y}}\right] E_{20}\right. \\
&+\left.\frac{1}{2}\left[-4 \sin (\Delta \psi)+\cos (\Delta \psi) \frac{\partial}{\partial \psi_{x}}\right] E_{11}\right\} \\
& L E_{\frac{1}{2} 1}=\epsilon\left\{\left[4 \sin (\Delta \psi)+\cos (\Delta \psi) \frac{\partial}{\partial \psi_{y}}\right] E_{11}\right. \\
&\left.+\frac{1}{2}\left[-2 \sin (\Delta \psi)+\cos (\Delta \psi) \frac{\partial}{\partial \psi_{x}}\right] E_{02}\right\}
\end{aligned}
$$

where $\Delta \psi=\psi_{x}-2 \psi_{y}$. To solve these equations, one must truncate this chain of equations at a certain order. For a small beam size $\left(\sigma_{x, y}\right.$ small), the higher order terms of $E_{i j}$ are much smaller than the lower order one, and the truncation is justified. The numerical simulation has shown that, for an initial Gaussian distribution, the final state is approximately Gaussian also. As a consequence, Eq.(13) can be used to truncate the chain. It is still not easy, however, to solve the set of nonlinear partial differential equations (16)-(18). One further simplification can be accomplished by finding the return map for the moments by integrating Eqs.(16)-(18) over one turn. To order $\epsilon$, we use the unperturbed values of $E_{i j}$ in the right-hand sides of Eqs.(17) and (18), i.e., $\partial E_{i, j} / \partial \psi_{x, y}=0$ for $i+j=2$. Then Eqs.(17) and (18) can be easily solved. Substituting the solutions of Eqs.(17) and (18) into Eq.(16) we obtain the change in $\left\langle I_{x}\right\rangle$, to order $\epsilon^{2}$,

$$
-\epsilon^{2}\left(4\left\langle I_{x} I_{y}\right\rangle-\left\langle I_{y}^{2}\right\rangle\right) \frac{1-\cos \left(2 \pi\left(2 \gamma_{y}-\gamma_{x}\right)\right)}{2\left(2 \gamma_{y}-\gamma_{x}\right)^{2}} .
$$

Combining Eqs.(8), (13) and (19), we find the return map for the emittances, to order $\epsilon^{2}$,

$$
\begin{aligned}
& x_{n+1}=x_{n}-\epsilon^{2} y_{n}\left(2 x_{n}-y_{n}\right) \frac{1-\cos \left(2 \pi\left(2 \gamma_{y}-\gamma_{x}\right)\right)}{\left(2 \gamma_{y}-\gamma_{x}\right)^{2}} \\
& y_{n+1}=y_{n}+2 \epsilon^{2} y_{n}\left(2 x_{n}-y_{n}\right) \frac{1-\cos \left(2 \pi\left(2 \gamma_{y}-\gamma_{x}\right)\right)}{\left(2 \gamma_{y}-\gamma_{x}\right)^{2}}
\end{aligned}
$$

where $x_{n}=\left.\left\langle I_{x}\right\rangle\right|_{\theta=2 \pi n}$ and $y_{n}=\left.\left\langle I_{y}\right\rangle\right|_{\theta=2 \pi n}$. For a given initial Gaussian distribution, the evolution of the emittances can be calculated from this map approximately. The fixed point which corresponds to the stationary state is

$$
2\left\langle I_{x}\right\rangle-\left\langle I_{y}\right\rangle=0 \text {. }
$$

If $\left\langle I_{x, y}\right\rangle=\sigma_{x, y}$ initially, the stationary state is found from Eqs.(8) and (21) as

$$
\begin{aligned}
\left\langle I_{x}\right\rangle & =\frac{1}{4}\left(2 \sigma_{x}+\sigma_{y}\right), \\
\left\langle I_{y}\right\rangle & =\frac{1}{2}\left(2 \sigma_{x}+\sigma_{y}\right) .
\end{aligned}
$$

Near the resonance, i.e. $\left|2 \gamma_{y}-\gamma_{x}\right|<<1$, this stationary state is locally stable. It implies that for an initial Gaussian distribution with $\left.\left\langle I_{x, y}\right\rangle\right|_{t=0}$ not too far away from the stationary values, the emittances in the final state: will achieve this stationary value. The curve $(c)$ in Fir.l is the evolution of $\left\langle I_{x, y}\right\rangle$ calculated from the map (20) of $2 \gamma_{y}-\gamma_{x}=0$. Outside the transient period, the result is in a good agreement with the numerical simulation, curve (a). From Fig.2 it can be seen that, in the transicmt period, the particle distribution deviatcs substantially from Gaussian showing that the approximation used to obtain the map (20) is invalid. The small discrepancy between the stationary state of the map (20) and the final stite of the tracking is a reflection of a small deviation of the final distribution from Gaussian. Fig.2 shows that, in the final state,

$$
\left\langle I_{y}^{2}\right\rangle-2\left\langle I_{y}\right\rangle^{2} \simeq 0.2\left\langle I_{y}\right\rangle^{2}
$$

for this special case. Using Eq.(23) instead of Eq.(1:3), we find the stationary state to be

$$
2\left\langle I_{x}\right\rangle-1.1\left\langle I_{y}\right\rangle=0
$$

The curve $(d)$ in Fig.1 is calculated from this modified map. It has a better agreement with the tracking result.

Far away from the resonance, i.e. $\left|2 \gamma_{y}-\gamma_{x}\right| \gg 1$, the map (20) is reduced to a linear map: $x_{n}=x_{n-1}$ and $y_{n}=$ $y_{n-1}$. As shown in the tracking (curve (b) in Fig.1), the motions in two directions are completely decoupled and the particle distributions in two directions remain unchanged.

In the above calculation, the approximations we have adopted are equivalent to neglecting high order fluctuations. It is not easy to evaluate these fluctuations alt hough it is possible in principle. An alternative way is to expand the distribution function. We rewrite the distribution function as

$$
f\left(I_{x}, \psi_{x}, I_{y}, \psi_{y}, \theta\right)=e^{-g\left(I_{x}, \psi_{x}, I_{y}, \psi_{y}, \theta\right)} .
$$

For the Gaussian distribution, $g=c+I_{x} / \sigma_{x}+I_{y} / \sigma_{y}$. It is expected that $f$ is not too far away from a Gaussian distribution provided that the coupling constant $\epsilon$ and the beam size are sufficiently small. If this is true, $g$ may be expanded in the form

$$
g\left(I_{x}, \psi_{x}, I_{y}, \psi_{y}, \theta\right)=\sum_{i, j=0}^{\infty} A_{i j} I_{x}^{\frac{1}{2}} I_{y}^{j}
$$

where $A_{i j}=A_{i j}\left(\psi_{x}, \psi_{y}, \theta\right)$, and

$$
E_{\frac{1}{2} j}=-\frac{\partial}{\partial A_{i j}} \int e^{-g} d I_{x} d I_{y} .
$$

Eq.(6) could then be solved approximately by truncating the series for $g$.

\section{References}

1. P. A. Sturrock, Annals of Physics, 3, 113(1958).

2. S. Ohnuma and R. L. Gluckstern, IEEE Trans. N'ucl. Sci., NS-32, 2261(1985).

3. L. E. Reichl, A Modern Course in Statistical Phlysics (University of Texas Press, Austin, 1980). 


\section{DISCLAIMER}

This report was prepared as an account of work sponsored by an agency of the United States Government. Neither the United States Government nor any agency thereof, nor any of their employees, makes any warranty, express or implied, or assumes any legal liability or responsibility for the accuracy, completeness, or usefulness of any information, apparatus, product, or process disclosed, or represents that its use would not infringe privately owned rights. Reference herein to any specific commercial product, process, or service by trade name, trademark, manufacturer, or otherwise does not necessarily constitute or imply its endorsement, recommendation, or favoring by the United States Government or any agency thereof. The views and opinions of authors expressed herein do not necessarily state or reflect those of the United States Government or any agency thereof. 

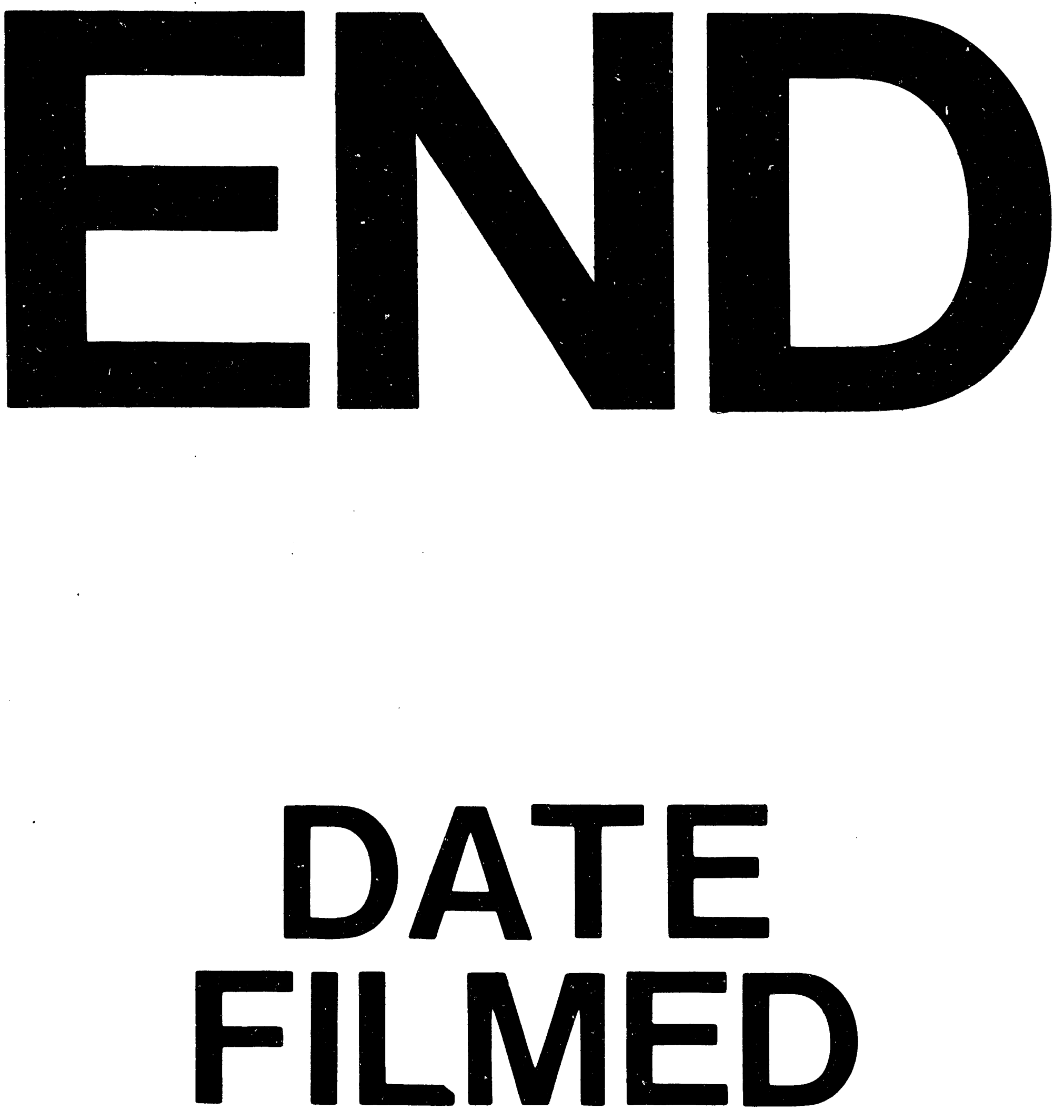

1

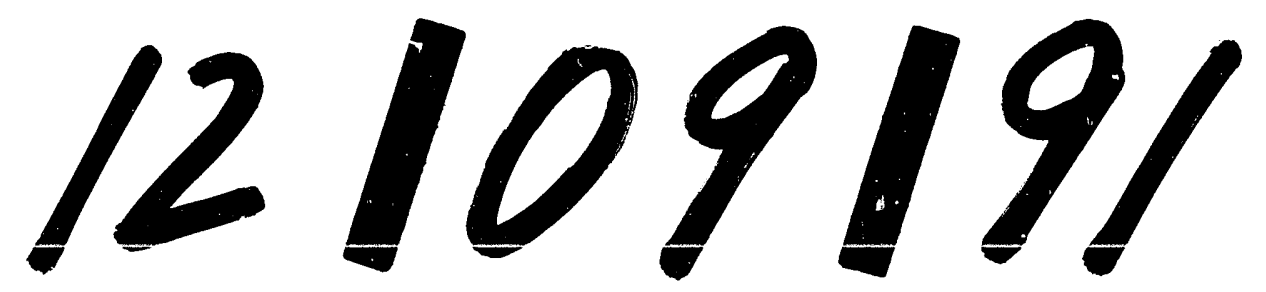


